\title{
A Flexible Bridge Rating Method Based on Analytical Evidential Reasoning and Monte Carlo Simulation
}

\author{
Saleh Abu Dabous ${ }^{1}{ }^{1}$ and Ghadeer Al-Khayyat ${ }^{2}$ \\ ${ }^{1}$ Department of Civil and Environmental Engineering, University of Sharjah, Sharjah, UAE \\ ${ }^{2}$ Sustainable Civil Infrastructure Systems Research Group, University of Sharjah, Sharjah, UAE \\ Correspondence should be addressed to Saleh Abu Dabous; sabudabous@sharjah.ac.ae
}

Received 18 February 2018; Revised 10 May 2018; Accepted 14 May 2018; Published 24 June 2018

Academic Editor: Dong Zhao

Copyright (C) 2018 Saleh Abu Dabous and Ghadeer Al-Khayyat. This is an open access article distributed under the Creative Commons Attribution License, which permits unrestricted use, distribution, and reproduction in any medium, provided the original work is properly cited.

\begin{abstract}
Several bridge inspection standards and condition assessment practices have been developed around the globe. Some practices employ four linguistic expressions to rate bridge elements while other practices use five or six, or adopt numerical ratings such as 1 to 9. This research introduces a condition rating method that can operate under different condition assessment practices and account for uncertainties in condition assessment by means of the Evidential Reasoning (ER) theory. The method offers flexibility in terms of using default elements and their weights or selecting alternative set of elements and condition rating schemes. The implemented ER approach accounts for uncertainties in condition rating by treating the condition assessments as probabilistic grades rather than numerical values. The ER approach requires the assignment of initial basic beliefs or probabilities, and typically these initial beliefs are assigned by an expert. Alternatively, this research integrates the Monte Carlo Simulation (MCS) technique with the ER theory to quantitatively estimate the basic probabilities and to produce robust overall bridge condition ratings. The proposed method is novel to the literature and has the following features: (1) flexible and can be used with any number of bridge elements and any standard of condition grades; (2) intuitive and simple paired comparison technique is implemented to evaluate weights of the bridge elements; (3) the MCS technique is integrated with the ER approach to quantify uncertainties associated with the stochastic nature of the bridge deterioration process; (4) the method can function with limited data and can incorporate new evidence to update the condition rating; (5) the final rating consists of multiple condition grades and is produced as a distributed probabilistic assessment reflecting the condition of the bridge elements collectively. The proposed method is illustrated with a real case study, and potential future research work is identified.
\end{abstract}

\section{Introduction}

Transportation bridges are critical to cities and societies as they facilitate movement of people and goods on daily basis. Bridge infrastructure is aging and deteriorating and requires attention either immediately or on the short term $[1,2]$. To address bridge infrastructure needs, several countries around the world developed and used bridge management systems (BMSs) to store and analyse bridge inventory data and to support the bridge management decision-making process in relation to budget allocation and the selection of efficient maintenance, repair, and replacement (MR\&R) interventions. The most important requirement of any BMS is the bridge condition assessment and rating module since condition rating relates directly to safety and serviceability of the structure.

Existing bridges are evaluated by either conducting an analytical evaluation of the load-carrying capacity or performing a visual inspection to rate the condition of the bridge elements. The analytical approach evaluates bridge elements load resistance compared to the applied service loads. The analytical evaluation and rating procedures are documented in codes of practice such as AASHTO LRFD [3], CAN/CSA-S6-14 [4], and ENV 1991-3 Eurocode 1 [5]. The other practices in bridge condition assessment are typically based on the bridge visual inspection process, during which experienced inspectors visit the bridge site to assess the condition of the various elements and assign 
subjective rating to each element based on extent and severity of identified defects and deterioration.

Several inspection manuals and condition assessment standards are developed by the various departments of transportation around the globe to regularize the bridge inspection and condition assessment process. These references provide procedures and guidelines to assist bridge inspectors in conducting the inspection and collecting the needed data. In addition, they include guidelines to assist in rating the bridge elements. Among these standards are the ones introduced by the Federal Highway Administration (FHWA) to manage bridges in the United States, including the National Bridge Inventory (NBI) and the National Bridge Inspection Standards [6]. The ministry of transportation of Ontario, Canada, developed the Ontario Structure Inspection Manual [7]. It provides descriptions and details of standard inspection procedures. In Europe, several projects had been undertaken to develop bridge inspection and maintenance standards such as Bridge Management in Europe (BRIME), Contecvet, Lifecon, and Rehabcon [8]. Condition assessment and rating methods capable of capturing data collected during bridge inspection and producing robust condition ratings are paramount for the bridge management process.

This research paper introduces a flexible bridge condition assessment and rating method that accounts for uncertainties associated with the visual inspection process. The approach can function with the different bridge inspection standards and requirements and uses paired comparison technique to intuitively assign weights to bridge elements. The method integrates the Monte Carlo simulation (MCS) with the evidential reasoning (ER) approach to quantify uncertainties associated with bridge assessment and rating processes.

\section{Literature Review}

Several studies aimed at developing bridge rating methods. Methods for component and system reliability are among the approaches that received acceptance for bridge evaluation [9]. The main purpose of the reliability assessment is to determine the load-carrying capacity of a bridge through estimation of a reliability index $\beta$. The main goal of the reliability analysis is to assess the bridge safety and eventually supporting the bridge management decision-making process regarding maintenance, retrofitting, and replacement interventions. Nowak et al. [10] compared reliability of concrete bridge girders designed based on guidelines of three different codes of practice. Zonta et al. [11] proposed a systematic reliability-based bridge management approach to assist in managing bridges in Italy. Estes and Frangopol [12] reviewed limitations and necessary modifications to condition rating practices and presented a study on how to use available visual inspection data to update reliability of bridges. They applied the approach to a superstructure of a deteriorating bridge in Colorado. Ghodoosi et al. [13] proposed a reliabilitybased deterioration model and demonstrated how to update the reliability of bridges using ground penetrating radar data. Estes and Frangopol [12] discussed that the reliability methods are powerful for quantifying risk and uncertainty, but they require a large amount of input data to perform the analysis. They also pointed to the fact that nondestructive testing and ground truth condition are needed inputs to perform reliability analysis, but these data are not available for every bridge.

Other studies focused on developing condition rating methods using available bridge inspection data and addressing subjectivity and uncertainty associated with the bridge visual inspection process. Researchers used fuzzy logic to address subjectivity associated with the use of linguistic expressions typically used for rating bridge elements. Tee et al. [14] were the first to propose a fuzzy weighted average approach. They developed membership functions to depict fuzziness in linguistic rating expressions. The data for the membership functions were collected through a questionnaire survey distributed to bridge engineers and inspectors from the state of Indiana and the neighbouring states. Further research was built on the principles of the fuzzy weighted average approach and introduced the integration of multiattribute decision-making techniques and fuzzy logic to rate concrete bridges $[15,16]$. A multilayer fuzzy synthesis assessment model to evaluate bridge damages was proposed in the literature. Five grades were selected for the multilayers: nondamage, light damage, moderate damage, severe damage, and unfit for service [17].

Artificial intelligence was investigated as a potential approach to enhance bridge condition assessment and condition rating. Li et al. [18] assessed the feasibility of using neural networks in bridge condition evaluation and proposed five subnets neural network for the deck, the superstructure, the substructure, the channel, and the overall evaluation. Cattan and Mohammadi [19] discussed that conventional statistical models and fuzzy-based methods were not successful in mapping between the subjective condition rating and the analytical evaluation of bridges. Alternatively, they used neural networks to map subjective ratings and bridge parameters and to compare subjective to analytical bridge ratings. Sobanjo [20] used neural networks to perform bridge condition rating. The network was trained to produce a condition rating based on inputting the age of a bridge in years. Case-based reasoning (CBR), a branch of artificial intelligence, was proposed in the literature to assess bridge deterioration and forecast future conditions. The underlying principle of the CBR is building a library of cases that can be used to solve a new problem if the new problem is similar to one of the cases in the database. Specific limitations of the CBR approach were identified, such as difficulties in retrieving similar cases especially if the library does not include sufficient cases, and complexity of developing a library of cases in a specific domain that can be used for case adaptation [21].

Wang and Elhag [22] reviewed fuzzy logic and neural network applications in bridge condition assessment and discussed that these approaches have limitations related to modelling uncertainties associated with the subjective ratings of bridge conditions. This can be attributed to the observation that fuzzy logic and neural networks may not provide a full description of the various levels of the overall assessment of a bridge structure. They discussed that the fuzzy inference model represents bridge condition rating and assumes that 
the condition ratings comply with the fuzzy number addition operation. In addition, the multilayer fuzzy synthesis assessment model requires bridge experts to subjectively assess each bridge element to single assessment grade with $100 \%$ confidence. They also discussed that the neural network models require significant amount of bridge inspection data to establish the mapping between inputs and outputs. Alternatively, Wang and Elhag [22] proposed the ER theory to model uncertainties inherent in bridge subjective assessments as an enhanced bridge condition assessment approach. The ER method produces distributed final overall assessment offering a panoramic view of the bridge condition ratings. They illustrated the ER method implementation with a hypothetical case study. Deng et al. [23] proposed the use of the D number with the ER method to perform bridge condition assessment and used Wang and Elhag's case study to demonstrate and validate their approach. Moufti et al. [24] proposed defect-based bridge condition assessment method based on the fuzzy hierarchal ER approach. They developed the needed membership functions based on defect severity levels defined by the Ministry of Transportation in Quebec.

Despite the significant effort to develop condition assessment and rating methods, further research is still needed. The literature review identified potential research areas that require further investigation, including (1) condition rating methods typically focus on one of the current practices in bridge condition assessment in term of selecting the assessment grades, choosing the breakdown of the bridge structure into elements, and selecting weights of these elements. A review of current practices (discussed in a later section) identified difficulties in standardizing bridge condition assessment process. In response, flexibility in condition assessment and rating methods needs to be enhanced; (2) the primary step in the ER method is defining the basic probability assignment (BPA). The BPA represents the confidence assigned to a certain proposition and reflects to what extent the existing evidence may support the proposition. The BPA values are usually assessed subjectively by an expert. A formal quantitative approach to estimate the BPA can enhance the process; and (3) the ER method uses either an analytical or a recursive algorithm to combine the various evidence. The literature lacks for studies to compare both algorithms performance when applied to bridge condition rating problem. This research proposes a flexible ER-based bridge condition rating method that attempt to address the above first and second limitations. Future work may expand on the current research to study and compare the performance of the analytical and the recursive algorithms.

\section{Bridge Inspection and Condition Assessment}

A thorough review of bridge inspection manuals shows that different countries in the world have implemented different bridge inspections and condition assessment guidelines. However, in principle, all these practices stem from the same method, which is the periodic visual inspection by welltrained teams of inspectors to assess conditions of the various bridge elements. Several initiatives in many countries in the world have been undertaken to develop and enhance bridge condition assessment practices. The FHWA established the National Bridge Inspection Standards to standardize the process and guide inspectors in reporting conditions of bridges on the public roadways [25]. The FHWA introduced a program to translate bridge condition data of commonly recognized bridge elements consistent with the National Bridge Inventory (NBI) condition ratings format [25]. The program helped the different states to report bridge inspection results to the NBI and enabled the use of the collected data in bridge management. The NBI rating system is based on a 0 to 9 scale to rate the elements while the final overall condition rating is reported in one of three categories: good, fair, and poor [3].

In Europe, a BMS known as BRIME (Bridge Management in Europe) was developed by the national highway research laboratories in the United Kingdom, France, Germany, Norway, Slovenia, and Spain [5]. The purpose of the project was to unify bridge management practices in these countries. Other European countries worked individually to set their bridge management standards. For instances, Denmark BMS uses bridge rating of $0-5$ to describe bridge damage [26], and the Swedish Road Administration uses a rating scale of 0-3 to describe the condition of the bridge [27]. In Canada, the Ministry of Transportation in Ontario developed and used its own structure inspection manual [4]. The manual adopts a four-level rating system: excellent, good, fair, and poor. For each element within the bridge, the inspector assesses and records deteriorated quantities of the different elements as an area, length, or unit based on the geometry and nature of the inspected element. The assessments are mainly developed based on inspector's visual observations and the use of some nondestructive testing to identify and quantify the extent of deterioration and defects. Then, the elements conditions are aggregated into an overall condition rating of the bridge structure. Table 1 shows some of the current inspection manuals and refers to the publishing agency and the rating system adopted by each manual.

The different bridge management practices varied in identifying recognized sets of bridge elements for inspection purposes and the weights assigned to these elements also varied from one practice to another. The AASHTO Guide Manual for Bridge Element Inspection introduced a complete and flexible set of bridge elements as an attempt to satisfy needs of the different departments of transportation in the United States. These elements were designed to capture all the components necessary to manage the needs of the NBI and to facilitate the utilization of the BMSs developed by the departments of transportation [28]. The various states in America selected specific sets of elements to be considered in their BMSs and assigned weights to reflect the relative importance of the elements. For example, Wang and Elhag [22] referred to the 13 bridge elements used in New York BMS and the assigned the weights as shown in Table 2.

The FHWA reviewed the shortcomings of the NBI and started an initiative to standardize data collection. They developed a "Commonly Recognized (CoRe) Structural Elements" manual, which included definition of elements and 
Table 1: Common North America and Europe bridge management systems with their bridge condition assessment.

\begin{tabular}{|c|c|c|c|}
\hline $\begin{array}{l}\text { Bridge management system } \\
\text { (manual) }\end{array}$ & Publishing & $\begin{array}{l}\text { Assessment grades } \\
\text { (rating system) }\end{array}$ & Meanings of the rating system \\
\hline $\begin{array}{l}\text { National bridge inventory } \\
\text { (NBI) [3] }\end{array}$ & US Department of Transportation & $0-9$ & $\begin{array}{l}\text { 0: failed condition; } 1 \text { : imminent } \\
\text { failure condition; } 2 \text { : critical } \\
\text { condition; } 3 \text { : serious condition; } \\
\text { 4: poor condition; } 5 \text { : fair condition; } \\
\text { 6: satisfactory condition; } 7 \text { : good } \\
\text { condition; } 8 \text { : very good condition; } \\
\text { 9: excellent condition. }\end{array}$ \\
\hline
\end{tabular}

New York BMS [22]

New York Road Department

$1-7$

Department of Transportation, State of Colorado

Bridge Ratings, Inspections and Records Manual (BRIAR) [27]

Manual [7]

\author{
Ontario Ministry of \\ Transportation
}

1: potentially hazardous; 3 : serious deterioration; 5: minor

deterioration; 7: excellent or new condition; 2, 4, and 6: between two adjacent ratings.

Poor: sufficiency rating less than 50 and status of structurally deficient or functionally obsolete. Fair: sufficiency rating from 50 to 80 and status of structurally deficient or functionally obsolete. Good: all remaining major bridges that do not meet the criteria for poor or fair.

Excellent: in as constructed condition

Good: first sign of minor defects is visible

Fair: medium defects are visible

Poor: severe defects are visible measurement units of 3-5 standardized condition grades, and suggested maintenance intervention for each condition grade. In 2010, the FHWA noticed specific issues associated with the implementation of this manual by the various departments of transportation, mainly related to inconsistencies in definition of condition grades and differences in the selected elements for inspection [25].

\section{Proposed Method for Bridge Condition Rating}

As discussed, bridge condition assessment is based on periodic visual inspection conducted by well-trained and experienced inspectors. However, the different practices adopted different condition assessment grades and specified different elements for inspection. The ER approach provides a suitable platform to develop a bridge condition assessment method due to its flexibility in incorporating any number of uncertain factors and ability to function with available limited data. Hence, the approach does not impose any limitations on the number of condition assessment grades or the number of bridge elements. In addition, the ER method is flexible to use different assessment grades for each element of the bridge if needed. The proposed method in this research utilizes paired comparison technique to enable the assessment of relative weights between the different bridge elements. The utilization of the paired comparison technique along with the ER method enables the application of the proposed method to any of the existing bridge condition
TABle 2: Bridge elements and weights used in New York BMS.

\begin{tabular}{lcc}
\hline Elements & Weights & Normalized weights \\
\hline 1. Bearing & 6 & 0.083 \\
2. Backwalls & 5 & 0.069 \\
3. Abutments & 8 & 0.111 \\
4. Wingwalls & 5 & 0.069 \\
5. Bridge seats & 6 & 0.083 \\
6. Primary members & 10 & 0.139 \\
7. Secondary members & 5 & 0.069 \\
8. Curbs & 1 & 0.014 \\
9. Sidewalks & 2 & 0.028 \\
10. Deck & 8 & 0.111 \\
11. Wearing surface & 4 & 0.056 \\
12. Piers & 8 & 0.111 \\
13. Joints & 4 & 0.56 \\
\hline
\end{tabular}

assessment practices or standards. The expert is required to specify the bridge elements, and then, the method can assess the relative weights through an intuitive pairwise comparison procedure. The proposed method introduces a new approach to assess the BPA quantitatively through the implementation of MCS. The final step in the proposed method is using the analytic ER algorithm to combine the basic probability assignments into an overall belief function reflecting the condition rating of the whole bridge structure. The sequence of the various steps of the proposed method is shown in Figure 1.

The following subsections explain the main steps of the proposed method. 


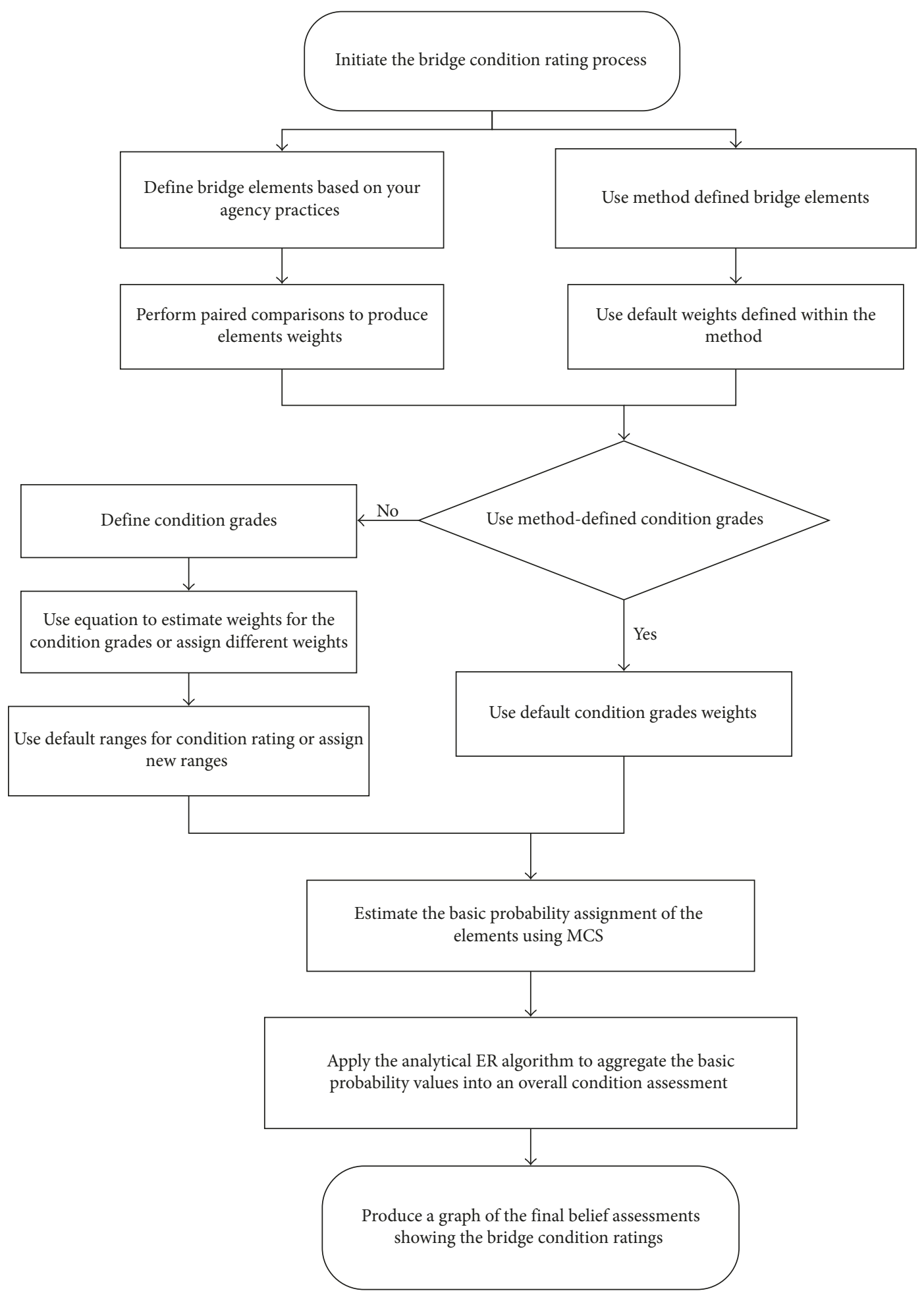

FIGURE 1: Flowchart for the condition assessment and rating method of concrete bridges.

4.1. Identifying Bridge Elements and Assigning Their Weights. Typically, any bridge consists of three major components: deck, superstructure, and substructure. In addition to these major components, several other elements are recognized. For instance, New York BMS breaks down the bridge into 13 elements as shown in Table 2. Examining the several bridge elements can be costly and is not always feasible due to limited resources and accessibility issues. The proposed method focuses on the three major components: deck, superstructure, and substructure. These are the default components included in the proposed method, as described in Figure 1. The components are divided into default elements for inspection and condition assessment purposes. At the same time, the method provides flexibility to revise the set of 
TABle 3: Paired comparisons for the bridge deck subelements provided by a bridge engineer.

A: wearing surface

\begin{tabular}{llllll} 
A & B: sidewalk & & & & \\
C & C & C: deck topside & & \\
D & D & C & D: deck underside & & \\
A & B/E & C & D & E: curbs & F: expansion joints \\
A $/ F$ & F & C & D & F & . \\
\hline
\end{tabular}

the default elements, so bridge engineer can identify specific important elements to be included in the assessment process.

Within the proposed method, the bridge engineer can either choose to use the standard weights assigned to the various elements used by their agency, such as the weights adopted by New York BMS (Table 2) or to produce the elements weights based on experience. In the latter case, paired comparisons can be used to extract the expert judgments and to evaluate the elements weight. The proposed approach is designed to be simple, intuitive, and flexible. To explain the approach, the bridge deck is used. The deck consists of six elements, namely: (1) the wearing surface, (2) the sidewalk, (3) the topside, (4) the underside, (5) the curbs, and (6) the expansion joints. The bridge engineer needs to fill each block in the matrix, such as the one provided in Table 3, to compare the two corresponding elements in pairs and to select the more important one. For instance, when the deck topside is compared to the wearing surface, the topside is more important, and the letter $\mathrm{C}$ is provided in the corresponding block in the matrix to reflect this judgment. If both elements have equal importance, then both elements are selected, such as the case in Table 3, where the bridge engineer assessed the expansion joints and the curbs to have the same level of importance.

The provided judgments can be translated into weights for the various elements depending on the frequency of selection of each element. The weight for each element is the frequency of the element selection divided by the total number of paired comparisons (15 in this case). For example, the wearing surface was selected 2.5 times (half time as one of the selections was shared with the expansion joints) so the weight is $2.5 / 15$ yielding a weight of 0.17 . Table 4 shows weights of the deck elements.

4.2. Selecting Assessment Grades and Assigning the Weights. As discussed, a variety of assessment grade and standards have been used in practice to rate the condition of bridge elements, including numeric rating scales such as 0 to 5 or 1 to 9 and linguistic expressions such as excellent, good, and poor. Our review of inspection practices (summarized in Table 1) shows that several manuals and condition rating methods adopted four or five main condition states and allowed for intermediate condition states to be defined as transition between the main states. Roberts and Shepard [29] developed a new index and proposed it as an improved numerical rating system that can assist in maintenance and repair decision-making. The index computations allow for selecting three, four, or five condition grades (State 1 to State 3, State 1 to State 4 , or State 1 to State 5). They provided
TABLE 4: Weights of the bridge deck subelements.

\begin{tabular}{lcc}
\hline Bridge element & Number of selections & Weight \\
\hline A & 2.5 & 0.17 \\
B & 0.5 & 0.03 \\
C & 5.0 & 0.33 \\
D & 4.0 & 0.27 \\
E & 0.5 & 0.03 \\
F & 2.5 & 0.17 \\
\hline
\end{tabular}

a formulation to assess the weights associated with any number of condition grades as in the following equation:

$$
\mathrm{WF}=\left[1-(\text { condition state\# }-1)\left(\frac{1}{\text { state count }}-1\right)\right] \text {. }
$$

For example, if the bridge inspector uses five condition states ranging from State 1 (brand new condition) to State 5 (worst condition), the weights are $1,0.75,0.5,0.25$, and 0 for condition States 1, 2, 3, 4, and 5, respectively. Ontario BMS adopted similar approach to the one developed by Roberts and Shepard, but the number of condition states was fixed to four (excellent, good, fair, and poor) and gave flexibility to the decision maker to assign the weights to these grades [30]. The proposed method here implements a default set of condition states and allows for using alternative condition states if necessary. The default condition states are excellent, good, fair, and poor, and the weights assigned to these four condition states are $0.9,0.7,0.45$, and 0.15 , respectively. These condition states and weights were recommended by bridge engineers during interview conducted to solicit their agency bridge management practices. At the same time, the proposed method provides flexibility to select different grades and weights.

4.3. ER Framework and Analytical Assessments Integration. The ER theory uses belief structure as an effective approach for modelling uncertainties since the structure has probabilistic nature and has the ability to function even in case of missing data or ignorance $[31,32]$. While implementing the ER theory, it is assumed that a frame of discernment $(\theta)$ is identified to include all the hypotheses under consideration where these hypotheses are exhaustive and mutually exclusive. Also, in this theory, it is assumed that with more evidence available, the hypothesis gets closer to the more precise possibility. The main concept in the ER theory is the BPA. The BPA is formally defined as the degree of belief in a subset of $\theta$ mapped over the interval $[0,1]$. The BPA is denoted by $m$, 
where $m(A)$ is the proportion of the total belief assigned to the subset $A$ of $\theta$. The BPA satisfies the following equations:

$$
\begin{aligned}
m(A) & \rightarrow[0,1], \\
m(\varnothing) & =0, \\
\sum_{A \subseteq \theta} m(A) & =1 .
\end{aligned}
$$

Another important concept in the ER theory is the traditional D-S rule of combination. This rule aggregates two basic probability assignments $m_{1}(B)$ and $m_{2}(C)$ into a joint probability $m_{1-2}(A)$ estimated as follows:

$$
m_{1-2}(A)=\frac{\sum_{B \cap C=A} m_{1}(B) m_{2}(C)}{\sum_{B \cap C \neq \varnothing} m_{1}(B) m_{2}(C)},
$$

where $A \neq \varnothing$ and $m_{1-2}(\varnothing)=0$.

To support the multiple attribute decision analysis requirements, the ER traditional rule of combination was further extended and two algorithms were developed, namely, the analytical and the recursive ER algorithms $[33,34]$. It is discussed in the literature that the analytical ER algorithm provides flexibility in aggregating a large number of attributes. In addition, its nonlinear features have been proven [22]. The analytical algorithm is implemented in this research.

The ER technique uses a distributed modelling framework to represent various quantitative and qualitative characteristics. Each characteristic is to be recognized with a set of assessment grades and a degree of uncertainty represented by a degree of belief. The distributed assessment model can evaluate element's condition with more than one grade, but the total degree of belief needs to sum up to 1 . For assessment integration purpose, the ER combination rule is utilized to aggregate the attributes of the various elements contributing to the overall condition assessment of the bridge structure [33]. Utilization of the ER approach for condition assessment starts with identification of the various elements that are part of the bridge and their weights that reflect their importance in the overall condition rating. Then, the basic probability masses (BPAs) of the various assessment grades for each element must be determined. After that, the analytical ER algorithm can be used for assessment aggregation of the different factors or elements. The frame of discernment considered in this study contains $\mathrm{N}$ assessment grades as follows:

$$
H=\left\{H_{\text {Poor }}, \ldots, H_{n}\right\},
$$

where $H_{\text {Poor }}$ is the worst condition grade and $H_{n}$ corresponds to the best condition state.

First step in the analytical ER is to convert the degrees of belief associated with the various assessment grades for each element to basic probability masses. The probability masses are calculated based on the relative weight of the element and the degree of belief in each assessment grade. It is assumed that the bridge structure includes I elements. Equations (5)-(15) below show analysis of the basic probability masses [35]: $m_{n, i}=m_{i}\left(H_{n}\right)=w_{i} \beta_{n, i}\left(a_{l}\right), \quad n=1, \ldots, N, i=1, \ldots, I$,

where $m_{n, i}$ is the basic probability mass of the bridge element, $w_{i}$ is the bridge element weight, and $\beta_{n, i}$ is the belief degree of the bridge element.

$$
\begin{array}{r}
m_{H, i}=m_{i}(H)=1-\sum_{n=1}^{N} m_{n, i}=1-w_{i} \sum_{n=1}^{N} \beta_{n, i}\left(a_{l}\right), \\
i=1, \ldots, I
\end{array}
$$

where $m_{H, i}$ is the probability mass assigned to the whole set $H$ and is divided into two parts, $\bar{m}_{H, i}$ and $\widetilde{m}_{H, i}$.

$$
\begin{aligned}
& \bar{m}_{H, i}=\bar{m}_{i}(H)=1-w_{i}, \quad i=1, \ldots, I, \\
& \widetilde{m}_{H, i}=\widetilde{m}_{i}(H)=w_{i}\left(1-\sum_{n=1}^{N} \beta_{n, i}\left(a_{l}\right)\right), \quad i=1, \ldots, I, \\
& m_{H, i}=\bar{m}_{H, i}+\widetilde{m}_{H, i} \text { and } \sum_{i=1}^{I} w_{i}=1,
\end{aligned}
$$

where $\bar{m}_{H, i}$ is produced by the relative importance of the bridge's elements and $\widetilde{m}_{H, i}$ is due to the incompleteness in bridge assessment.

The analytical ER equations can be used then used for aggregating the assessments obtained from the various elements of the bridge structure. Equations (10)-(15) shown below are used for computing the aggregated overall assessments [29]:

$$
\begin{array}{r}
\left\{H_{n}\right\}: m_{n}=k\left[\prod_{i=1}^{I}\left(m_{n, i}+\bar{m}_{H, i}+\tilde{m}_{H, i}\right)-\prod_{i=1}^{I}\left(\bar{m}_{H, i}+\tilde{m}_{H, i}\right)\right], \\
n=1, \ldots, N
\end{array}
$$

where $k$ is the normalization factor of the analytical ER algorithm.

$$
\begin{aligned}
\{H\}: \tilde{m}_{H} & =k\left[\prod_{i=1}^{I}\left(\bar{m}_{H, i}+\tilde{m}_{H, i}\right)-\prod_{i=1}^{I} \bar{m}_{H, i}\right], \\
\{H\}: \bar{m}_{H} & =k\left[\prod_{i=1}^{I} \bar{m}_{H, i}\right], \\
k & =\left[\sum_{n=1}^{N} \prod_{i=1}^{I}\left(m_{n, i}+\bar{m}_{H, i}+\widetilde{m}_{H, i}\right)\right. \\
& \left.-(N-1) \prod_{i=1}^{I}\left(\bar{m}_{H, i}+\widetilde{m}_{H, i}\right)\right]^{-1}, \\
\left\{H_{n}\right\} & : \beta_{n}=\frac{m_{n}}{1-\bar{m}_{H}}, \quad n=1, \ldots, N,
\end{aligned}
$$




$$
\{H\}: \beta_{H}=\frac{\widetilde{m}_{H}}{1-\bar{m}_{H}},
$$

where $\beta_{n}$ and $\beta_{H}$ stand for the overall belief degrees of the aggregated assessments assigned to the assessment grades $H_{n}$ and $H$, respectively.

Equations (14) and (15) above are used for normalization purpose of the combined probability assignments into overall belief degrees.

\subsection{Quantifying the Basic Probability Assignment Using} Monte Carlo Simulation. The BPA is a fundamental concept of the ER theory. It reflects the degree of belief in a subset of the frame of discernment. In case of bridge condition assessment, the frame of discernment contains all the possible condition states that an inspector can rate the bridge elements with. The total belief is proportionally mapped to the different elements by assigning a BPA to each bridge element reflecting the element condition rating. Then, the ER rule of combination (the core of the ER theory) is applied to combine the different BPA values. As discussed, the BPA is typically assigned by an expert. The expert assessments are subjective and can suffer from inconsistencies inherent in implementing human judgments and experience. Two different experts may review the same inspection report and assign two different probabilities to the reported condition rating of an element. Alternatively, the proposed method uses the MCS technique to quantitatively assess the BPA value based on the data reported in inspection reports.

The MCS is a stochastic technique to quantify uncertainties in a system or a model. The technique generates several possible scenarios by running any computational algorithm for hundreds or thousands of iterations to produce several possible outcomes. In each iteration, the simulation follows a standard procedure to generate random values of the uncertain variables. Then, all the generated possible outcomes are produced as a probability distribution with statistical analysis of the results. From the probability distribution, the probability of having the outcomes within any possible range of results can be identified.

A condition index model is needed to run the MCS and to evaluate probabilities of the different condition grades. The concept of health index (HI) implemented by Department of Transportation in California [29] is adopted for this purpose. The HI is based on assessing the remaining asset value of a bridge or network of bridges using bridge inspection results. Its rationale is that the asset value of a bridge element drops as the element deteriorates with time to lower condition states. On the contrary, the element asset value increases when maintenance or rehabilitation actions are performed to enhance the element condition rating. Building on this concept, a bridge element HI can be estimated as a weighted average of the different quantities of the elements and their condition states as follows:

$$
\mathrm{HI}=\frac{\sum_{i}^{n} Q_{i} W_{i}}{Q_{\text {total }} W_{\text {new }}} \times 100,
$$

\begin{tabular}{|c|c|c|c|}
\hline \multicolumn{2}{|l|}{ Component } & Element & Weights \\
\hline \multirow{6}{*}{ Deck (0.33) } & & Wearing surface & 0.17 \\
\hline & & Sidewalk & 0.03 \\
\hline & & Deck topside & 0.33 \\
\hline & & Deck underside & 0.27 \\
\hline & & Curbs & 0.03 \\
\hline & & Expansion joints & 0.17 \\
\hline \multirow{5}{*}{$\begin{array}{l}\text { Superstructure } \\
(0.33)\end{array}$} & & Strings & 0.30 \\
\hline & & Floor beams & 0.30 \\
\hline & & $\begin{array}{l}\text { Floor system } \\
\text { bracing }\end{array}$ & 0.05 \\
\hline & & Girders & 0.30 \\
\hline & & Bearing devices & 0.05 \\
\hline \multirow{9}{*}{$\begin{array}{l}\text { Substructure } \\
(0.33)\end{array}$} & \multirow{6}{*}{$\begin{array}{l}\text { Abutments } \\
(0.50)\end{array}$} & Bearing seats & 0.04 \\
\hline & & Backwall & 0.33 \\
\hline & & Wingwalls & 0.33 \\
\hline & & Piles & 0.15 \\
\hline & & Footing & 0.15 \\
\hline & & Piles & 0.42 \\
\hline & \multirow{3}{*}{ Piers $(0.50)$} & Footing & 0.42 \\
\hline & & Columns & 0.08 \\
\hline & & Caps & 0.08 \\
\hline
\end{tabular}

TABle 5: Paired comparison of elements structural importance.

where $Q_{i}$ is the quantity of the element inspected and assessed to be in the condition state $i, W_{i}$ is the weight assigned to the condition state $i, Q_{\text {total }}$ is the total quantity of the bridge element, and $W_{\text {new }}$ is the weight assigned to the element when it was in a relatively new condition. As an example, the inspection report indicated that $100 \mathrm{~m}^{2}$ of the girders surface area is rated as good and $80 \mathrm{~m}^{2}$ is rated as fair, and then using the default weights, the $\mathrm{HI}$ is calculated as follows:

$$
\mathrm{HI}=\frac{(0 \times 0.9+100 \times 0.7+80 \times 0.45+0 \times 0.15)}{(0.9 \times 180)} \times 100=65 \text {. }
$$

The estimated HI can be assigned to a condition state by specifying a range of $\mathrm{HI}$ for each condition state. The proposed ranges for the different condition states are assigned to be consistent with the weight of each condition grade. For example, if the $\mathrm{HI}$ is above 85 , then the element is in excellent condition; if less than 45 , then the element is in poor condition; while 65 to 85 is good; and 45 to 65 is fair. In the above example, the bridge beams are exactly at the border point between good and fair condition states, and it is not clear how to rate them. Hence a more sensitive approach to quantify risk is needed.

The MCS can be implemented on this model to estimate the BPA values by building several scenarios for the HI while varying the weights assigned to the different condition ratings. The simulation technique accounts for uncertainty in defining the weights and provides stochastic analysis for the HI. The frequency of having the condition index in each condition state range estimates the probability of having the element in that condition state. The MCS approach can quantitatively estimate the basic probabilities of the different condition ratings to be assigned to each element, which assists in eliminating the subjectivity associated with the 


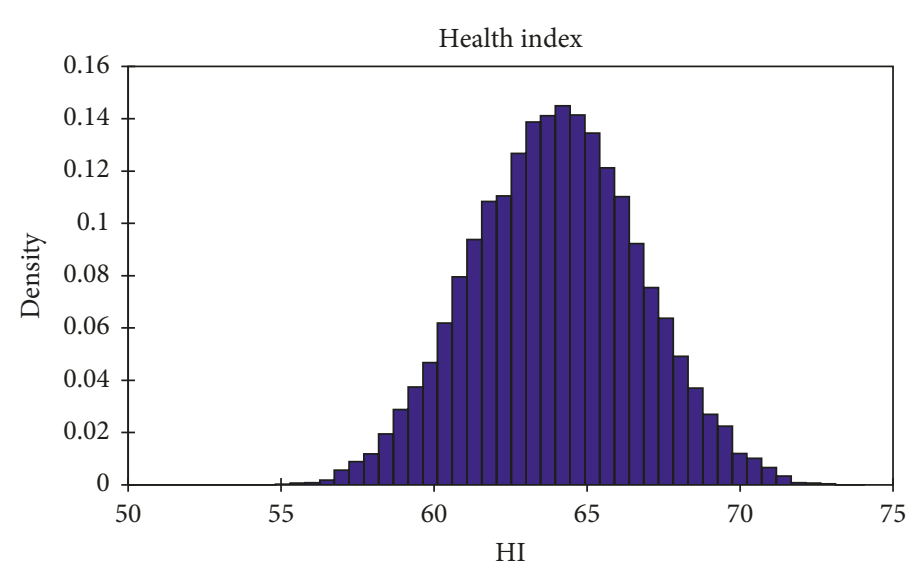

\begin{tabular}{lc}
\hline Statistic & HI \\
\hline $\begin{array}{l}\text { Number of } \\
\text { observations }\end{array}$ & 10000 \\
Minimum & 55.255 \\
Maximum & 73.079 \\
Range & 17.824 \\
Median & 64.011 \\
Mean & 64.000 \\
Variance ( $n)$ & 7.381 \\
Standard & 2.717 \\
deviation ( $n$ ) & \\
Variation & 0.042 \\
coefficient & \\
Skewness (Pearson) & 0.009 \\
Kurtosis (Pearson) & -0.230 \\
Geometric mean & 63.942 \\
Geometric deviation & 1.043 \\
\hline
\end{tabular}

FIGURE 2: The MCS results for the bridge deck.

TABLE 6: Bridge elements distributed assessments.

\begin{tabular}{|c|c|c|c|}
\hline Component & & Element & Distributed assessment (BPA) \\
\hline Deck & & $\begin{array}{l}\text { Wearing surface } \\
\text { Sidewalk } \\
\text { Deck topside } \\
\text { Deck underside } \\
\text { Curbs } \\
\text { Expansion joints }\end{array}$ & $\begin{array}{l}\{(\text { Poor, 0), (Fair, 0.30), (Good, 0.70), (Excellent, 0)\} } \\
\{(\text { Poor, 0), (Fair, 0.20), (Good, 0.80), (Excellent, 0) }\} \\
\{(\text { Poor, 0), (Fair, 0.25), (Good, 0.75), (Excellent, 0) }\} \\
\{(\text { Poor, 0), (Fair, 0.40), (Good, 0.60), (Excellent, 0) }\} \\
\{(\text { Poor, 0), (Fair, 0.10), (Good, 0.90), (Excellent, 0) }\} \\
\{(\text { Poor, 0), (Fair, 0.15), (Good, 0.85), (Excellent, 0) }\}\end{array}$ \\
\hline Superstructure & & $\begin{array}{l}\text { Strings } \\
\text { Floor beams } \\
\text { Floor system bracing } \\
\text { Girders } \\
\text { Bearing devices }\end{array}$ & $\begin{array}{l}\{(\text { Poor, 0.70), (Fair, 0.30), (Good, 0), (Excellent, 0) }\} \\
\{(\text { Poor, 0.90), (Fair, 0.10), (Good, 0), (Excellent, 0) } \\
\{(\text { Poor, 0.40), (Fair, 0.60), (Good, 0), (Excellent, 0) }\} \\
\{(\text { Poor, 0.80), (Fair, 0.20), (Good, 0), (Excellent, 0) }\} \\
\{(\text { Poor, 0.40), (Fair, 0.60), (Good, 0), (Excellent, 0) }\}\end{array}$ \\
\hline Substructure & Abutments & $\begin{array}{l}\text { Bearing seats } \\
\text { Backwall } \\
\text { Wingwalls } \\
\text { Piles } \\
\text { Footing } \\
\text { Piles } \\
\text { Footing } \\
\text { Columns } \\
\text { Caps }\end{array}$ & $\begin{array}{l}\{(\text { Poor, 0.20), (Fair, 0.50), (Good, 0.30), (Excellent, } 0)\} \\
\{(\text { Poor, 0.30), (Fair, 0.40), (Good, 0.30), (Excellent, 0) }\} \\
\{(\text { Poor, 0.50), (Fair, 0.30), (Good, 0.20), (Excellent, 0) }\} \\
\{(\text { Poor, 0), (Fair, 0.80), (Good, 0.20), (Excellent, 0) } \\
\{(\text { Poor, 0.10), (Fair, 0.70), (Good, 0.20), (Excellent, 0) }\} \\
\{(\text { Poor, 0), (Fair, 0.60), (Good, 0.40), (Excellent, 0) }\} \\
\{(\text { Poor, 0), (Fair, 0.80), (Good, 0.20), (Excellent, 0) }\} \\
\{(\text { Poor, 0.60), (Fair, 0.40), (Good, 0), (Excellent, 0) }\} \\
\{(\text { Poor, 0), (Fair, 0.80), (Good, 0.20), (Excellent, 0) }\}\end{array}$ \\
\hline
\end{tabular}

direct assignment through expert judgment. These basic probabilities can then be input into the ER algorithms to combine them and generate the overall bridge condition rating.

\section{Case Study}

The proposed method in this research is demonstrated with a case study. The data for the case study is extracted from bridge inspection report. The condition ratings used by the inspector are the four standard condition grades utilized in the proposed method which are poor, fair, good, and excellent conditions. A bridge engineer was requested to analyse the inspection report and to submit the needed paired comparisons to assess the elements weight. The technique is illustrated in the proposed method section, and the weights of the various elements of the bridge are shown below in Table 5 .
To assess the probabilities associated with the different condition grades, the MCS is performed. The XL Stat software is used to run 10,000 iterations for each bridge element. The simulation assigns random values for the different condition grades to represent the weights associated with the corresponding condition grades. Then, the $\mathrm{HI}$ is estimated as per (16). This process is repeated 10,000 times, and the final statistics are produced by the software in form of tables and graphs. Figure 2 shows the MCS results for the bridge deck. The frequency of having the HI in each condition grade compared to the total number of iterations reflects the chance of having the element in the corresponding condition rating. For instance, for the bridge deck, around 2,500 iterations produced an $\mathrm{HI}$ in the range of fair and 7,500 iterations produced $\mathrm{HI}$ in the range of a good condition state. As a result, the bridge deck topside had 
TABLE 7: Basic probability masses of the piers elements considering the various assessment grades.

\begin{tabular}{lccccccc}
\hline \multirow{2}{*}{ Substructure component } & \multicolumn{3}{c}{ Grade/basic probability mass } & & \\
& \multicolumn{2}{c}{$m_{n, i}$} & Good & Excellent & $m_{H, i}$ & $\bar{m}_{H, I}$ \\
\hline Piles & Poor & Fair & 0.252 & 0.168 & 0 & 0.580 & 0.580 \\
Footing & 0 & 0.336 & 0.084 & 0 & 0.580 & 0.580 \\
Columns & 0.048 & 0.032 & 0.000 & 0 & 0.920 & 0.920 \\
Caps & 01 & 0.064 & 0.016 & 0 & 0.920 & 0.920 \\
\hline
\end{tabular}

a $25 \%$ chance of receiving fair rating and $75 \%$ chance of receiving good rating.

The MCS is performed for all the bridge elements, and the basic probabilities produced are shown in Table 6 .

Since the BPA values and elements weights are determined, the ER analysis can be performed. The ER analytical algorithm can be applied for assessment aggregation and overall beliefs calculation. The details of the calculations are illustrated with a sample numerical example for the assessment of the bridge substructure. The steps below show the calculation process for the degrees of belief, which illustrate the main steps needed to estimate the overall condition of the bridge. Substructure component with its abutments and piers elements is considered for calculation demonstration as shown below. The Piles element from the piers substructure component is considered first with the following distributed assessment and weight:

(i) Distributed assessment: $\{($ Poor, 0), (Fair, 0.60), (Good, 0.40), (Excellent, 0)\}

(ii) Weight: 0.42

Step 1. Calculate the basic probability masses of the bridge element in the various assessment grades:

$$
\text { (i) } \begin{aligned}
& m_{\text {Fair,Piles }}=m_{\text {Piles }}\left(H_{\text {Fair }}\right)=w_{\text {Piles }} \times \beta_{\text {Fair,Piles }} \\
&=0.42(0.60)=0.252 \\
& \text { (ii) } \begin{aligned}
m_{\text {Good,Piles }} & =m_{\text {Piles }}\left(H_{\text {Good }}\right)=w_{\text {Piles }} \times \beta_{\text {Good, Piles }} \\
& =0.42(0.40)=0.168
\end{aligned}
\end{aligned}
$$

Step 2. Calculate the probability mass assigned to the whole set $\mathrm{H}$ :

$$
\begin{aligned}
& \text { (i) } m_{H \text {, Piles }}=m_{\text {Piles }}(H)=1-\sum_{n=1}^{4} m_{n} \text {, Piles } \\
& =1-w_{\text {Piles }} \sum_{n=1}^{4} \beta_{n \text {, Piles }} \\
& =1-(0+0.252+0.168+0)=0.580 \\
& \text { (ii) } \bar{m}_{H \text {, Piles }}=\bar{m}_{\text {Piles }}(H)=1-w_{\text {Piles }}=1-0.42=0.580 \\
& \text { (iii) } \widetilde{m}_{H \text {, Piles }}=\widetilde{m}_{\text {Piles }}(H)=w_{\text {Piles }}\left(1-\sum_{n=1}^{4} \beta_{n \text {,Piles }}\right) \\
& =0.42(1-(0+0.60+0.40+0))=0 \\
& \text { (iv) } m_{H, \text { Piles }}=\bar{m}_{H \text {,Piles }}+\widetilde{m}_{H \text {,Piles }}=0.580+0=0.580
\end{aligned}
$$

Steps 1 and 2 are repeated for the other elements in the piers of the substructure bridge component. The data in Table 7 show the grade probability distribution of the various elements.

Step 3. Aggregate the basic probability masses from the various elements for each assessment grade:

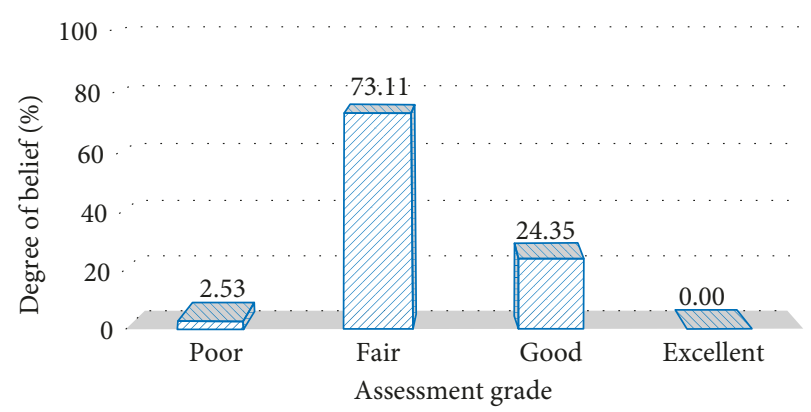

Figure 3: Overall condition assessment of the piers element of the substructure bridge component.

(i) $\begin{aligned} k= & {\left[\sum_{n=1}^{4} \prod_{i=1}^{4}\left(m_{n, i}+\bar{m}_{H, i}+\tilde{m}_{H, i}\right)\right.} \\ & \left.-(N-1) \prod_{i=1}^{4}\left(\bar{m}_{H, i}+\widetilde{m}_{H, i}\right)\right]^{-1} \\ & =((1.726)-(4-1)(0.285))^{-1}=1.148\end{aligned}$

(ii) $\left\{H_{n}\right\}: m_{\text {Poor }}=k\left[\prod_{i=1}^{4}\left(m_{n, i}+\bar{m}_{H, i}+\widetilde{m}_{H, i}\right)\right.$

$$
\left.-\prod_{i=1}^{4}\left(\bar{m}_{H, i}+\widetilde{m}_{H, i}\right)\right]
$$$$
=1.148(0.300-0.285)=0.017
$$

(iii) $\left\{H_{n}\right\}: m_{\text {Fair }}=k\left[\prod_{i=1}^{4}\left(m_{n, i}+\bar{m}_{H, i}+\widetilde{m}_{H, i}\right)\right.$

$$
\left.-\prod_{i=1}^{4}\left(\bar{m}_{H, i}+\widetilde{m}_{H, i}\right)\right]
$$$$
=1.148(0.714-0.285)=0.492
$$

(iv) $\left\{H_{n}\right\}: m_{\mathrm{Good}}=k\left[\prod_{i=1}^{4}\left(m_{n, i}+\bar{m}_{H, i}+\tilde{m}_{H, i}\right)\right.$

$$
\begin{aligned}
& \left.-\prod_{i=1}^{4}\left(\bar{m}_{H, i}+\widetilde{m}_{H, i}\right)\right] \\
& =1.148(0.428-0.285)=0.164
\end{aligned}
$$

(v) $\left\{H_{n}\right\}: m_{\text {Excellent }}=k\left[\prod_{i=1}^{4}\left(m_{n, i}+\bar{m}_{H, i}+\widetilde{m}_{H, i}\right)\right.$

$$
\begin{aligned}
& \left.-\prod_{i=1}^{4}\left(\bar{m}_{H, i}+\tilde{m}_{H, i}\right)\right] \\
& =1.148(0.285-0.285)=0
\end{aligned}
$$

(vi) $\{H\}: \widetilde{m}_{H}=k\left[\prod_{i=1}^{4}\left(\bar{m}_{H, i}+\widetilde{m}_{H, i}\right)-\prod_{i=1}^{4} \bar{m}_{H, i}\right]$

$$
=1.148(0.285-0.285)=0
$$

(vii) $\{H\}: \bar{m}_{H}=k\left[\prod_{i=1}^{4} \bar{m}_{H, i}\right]=1.148(0.285)=0.327$

Step 4. Normalize the combined probability assignments into overall belief degrees:

(i) $\left\{H_{n}\right\}: \beta_{\text {Poor }}=m_{\text {Poor }} / 1-\bar{m}_{H}$

$$
=0.017 / 1-0.327=0.025
$$

(ii) $\left\{H_{n}\right\}: \beta_{\text {Fair }}=m_{\text {Fair }} / 1-\bar{m}_{H}$

$$
=0.492 / 1-0.327=0.731
$$


TABLE 8: Basic probability masses of the abutments elements considering the various assessment grades.

\begin{tabular}{|c|c|c|c|c|c|c|c|}
\hline \multirow{3}{*}{ Substructure component } & \multicolumn{4}{|c|}{ Grade/basic probability mass } & \multirow{3}{*}{$m_{H, i}$} & \multirow{3}{*}{$\bar{m}_{H, I}$} & \multirow{3}{*}{$\tilde{m}_{H, I}$} \\
\hline & \multicolumn{4}{|c|}{$m_{n, i}$} & & & \\
\hline & Poor & Fair & Good & Excellent & & & \\
\hline Bearing seats & 0.008 & 0.020 & 0.012 & 0 & 0.960 & 0.960 & 0 \\
\hline Backwall & 0.099 & 0.132 & 0.099 & 0 & 0.670 & 0.670 & 0 \\
\hline Wingwalls & 0.165 & 0.099 & 0.066 & 0 & 0.670 & 0.670 & 0 \\
\hline Piles & 0 & 0.120 & 0.030 & 0 & 0.850 & 0.850 & 0 \\
\hline Footing & 0.015 & 0.105 & 0.030 & 0 & 0.850 & 0.850 & 0 \\
\hline
\end{tabular}

(iii) $\left\{H_{n}\right\}: \beta_{\mathrm{Good}}=m_{\mathrm{Good}} / 1-\bar{m}_{H}$

$$
=0.164 / 1-0.327=0.244
$$

(iv) $\left\{H_{n}\right\}: \beta_{\text {Excellent }}=m_{\text {Excellent }} / 1-\bar{m}_{H}$

$$
=0 / 1-0.327=0
$$

(v) $\{H\}: \beta_{H}=\widetilde{m}_{H} / 1-\bar{m}_{H}=0 / 1-0.327=0$

The data in Figure 3 show the overall condition assessment of the piers element of the bridge. However, for overall condition assessment of the substructure component, Steps 1 to 4 need to be repeated for the abutments elements.

Table 8 shows the basic probability masses of the abutments elements. After performing Steps 3 and 4, the normalized overall belief degrees of the abutments element are found to be the following: $\{($ Poor, 0.29), (Fair, 0.49), (Good, 0.23), (Excellent, 0)\}.

The overall condition assessments of the piers and abutments are combined for overall substructure condition assessment (Figure 4). The same steps are preformed on the bridge deck and bridge superstructure to find their overall conditions. Then, the condition assessment of the bridge deck, superstructure, and substructure are aggregated to assess the overall condition of the inspected bridge. Overall, the bridge was assessed to be in poor, fair, and good conditions with $26.93 \%, 42.66 \%$, and $30.41 \%$ probability, respectively, as shown in Figure 5.

The obtained combined distributed assessment is a definite enhancement over evaluating the bridge elements individually or evaluating the overall condition with a single numerical index. The bridge deck, a major element of the bridge structure, and its elements in this case are in good conditions, which individually may give a misleading assessment of the bridge overall condition and the urgency of maintenance needs. However, the elements of the bridge superstructure are mostly in poor condition, impacting the overall condition of the bridge, which clearly indicates that the bridge requires maintenance intervention.

\section{Summary and Conclusions}

Based on reviewing bridge inspection manuals and practices, it is clearly noticed that inspection standards and guidelines vary from one country to another and may even vary in the different states or districts within the same country, despite the fact most of the practices rely mostly on the same approach of visual inspection for data collection. In addition, it is widely reported in the literature that results obtained from bridge visual inspection are inevitably uncertain and

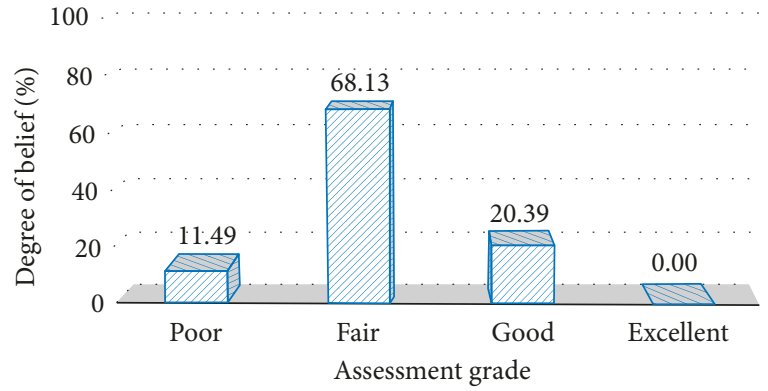

FIGURE 4: Overall condition assessment of the substructure bridge component.

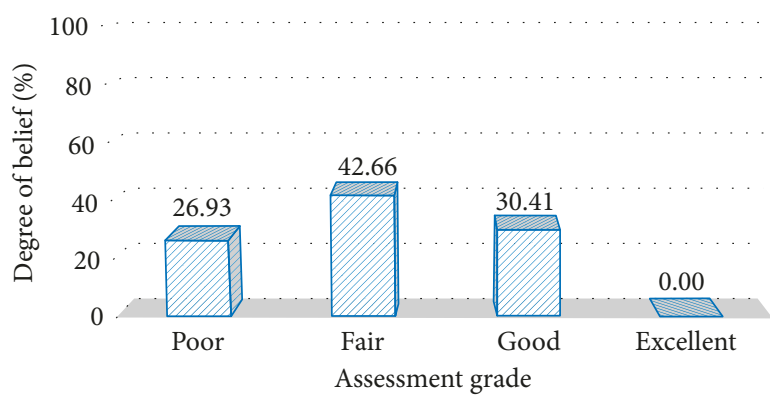

FIGURE 5: Overall bridge condition assessment based on the analytical ER algorithm.

subjective. The current research contributes a flexible condition rating method that can function with different bridge inspection standards and guidelines and account for uncertainties inherent in the visual inspection process. Generally, uncertainties in the bridge condition assessment are addressed using either fuzzy logic or probabilistic analysis. The fuzzy logic quantifies subjectivity associated with using language expressions for condition ratings while probabilistic analysis focuses on randomness and uncertainties associated with quantifying the condition rating. The bridge deterioration process is stochastic in nature, and probabilistic analysis can better depict stochastic behaviour. The proposed method uses the analytical ER approach that treats condition assessments as probabilistic assessment grades. The method integrates the MCS technique with the ER approach to enhance bridge condition rating by eliminating subjectivity in assessing the initial degrees of belief. The MCS method quantitatively estimates the BPA values that are aggregated by means of the recursive ER algorithm to 
produce a robust overall condition rating of the bridge structure.

The proposed method can enhance bridge condition assessment and rating processes because of the following reasons: (1) flexibility in aggregating any number of basic probabilities assigned to any number of bridge elements and condition grades; (2) flexibility in defining the bridge elements and assigning the weights. The weights can be used as the default weights defined within the proposed method or assessed by the decision maker through the intuitive paired comparison technique; (3) the approach allows for incorporating new evidence as new inspection records become available; and (4) both the MCS and the ER are probabilistic techniques that are suitable and consistent in modelling uncertainties associated with the bridge condition assessment process.

The proposed method requires the expert input in certain cases, especially if the expert decided to use different parameters other than the ones embedded within the framework, such as when the expert decides to revise the weights of the bridge elements or condition grades. In this case, subjective assessments are needed. The intuitive paired comparison technique is implemented to assist the expert in estimating the different elements weights in a systematic way. Further work to study the uncertainty in the assigned weights to the different elements can enhance the proposed method. Other future research directions can include (1) to study the implementation of the recursive ER algorithm in bridge condition assessment and to compare its results with the results obtained from the analytical ER algorithm, (2) to compare the probabilistic ER analysis with the fuzzy-based methods, and (3) to apply the proposed method on more case studies and other civil engineering applications.

\section{Conflicts of Interest}

The authors declare that they have no conflicts of interest.

\section{Acknowledgments}

This research was funded by the Sustainable Civil Infrastructure Systems Research Group working under the Research Institute of Sciences and Engineering (RISE), University of Sharjah. The authors would like to thank Engineer Daniel LIort from the Ministry of Infrastructure Development in the United Arab Emirates for his help in providing judgments needed for the analysis of the case study.

\section{References}

[1] ASCE, Report Card for America's Infrastructure, American Society of Civil Engineers, 2017, http://www.infrastructurereportcard.org.

[2] CIRC, Report Card for Canada's Infrastructure, 2016, http:// www.canadainfrastructure.ca.

[3] AASHTO, LRFD Bridge Design Specifications, American Association of State Highway and Transportation Officials, Washington, DC, USA, 2007.

[4] CSA, Canadian Highway Bridge Design Code (Public Review Version), CSA International, Mississauga, Ontario, Canada, 2013.
[5] Eurocode ENV (1991-3), Eurocode 1: Basis of Design and Actions on Structures. Part 3: Traffic Loads on Bridges, 1994.

[6] K. F. Dunker and B. G. Rabbat, "Assessing infrastructure deficiencies: the case of highway bridges," Journal of Infrastructure Systems, vol. 1, no. 2, pp. 100-119, 1995.

[7] OSIM, Ontario Structure Inspection Manual, Ontario Ministry of Transportation, Policy, Planning \& Standards Division, Engineering Standards Branch, Bridge Office, St. Catharines, ON, Canada, 2008, https://www.ogra.org/files/ OSIM\%20April\%202008.pdf.

[8] R. Helmerich, E. Niederleithinger, D. Algernon, D. Streicher, and $\mathrm{H}$. Wiggenhauser, "Bridge inspection and condition assessment in Europe," Transportation Research Record: Journal of the Transportation Research Board, vol. 2044, pp. 31-38, 2008.

[9] D. M. Frangopol, M. Ghosn, G. Hearn, and A. Nowak, "Structural reliability in bridge engineering," Journal of Bridge Engineering, vol. 3, no. 4, pp. 151-154, 1998.

[10] A. S. Nowak, C. H. Park, and J. R. Casas, "Reliability analysis of prestressed concrete bridge girders: comparison of Eurocode, Spanish Norma IAP and AASHTO LRFD," Structural Safety, vol. 23, no. 4, pp. 331-344, 2001.

[11] D. Zonta, R. Zandonini, and F. Bortot, "A reliability-based bridge management concept," Structures and Infrastructure Engineering, vol. 3, no. 3, pp. 215-235, 2007.

[12] A. C. Estes and D. M. Frangopol, "Updating bridge reliability based on bridge management systems visual inspection results," Journal of Bridge Engineering, vol. 8, no. 6, pp. 374-284, 2003.

[13] F. Ghodoosi, A. Bagchi, and T. Zayed, "Method for developing and updating deterioration models for concrete bridge decks using GPR data," Automation in Construction, vol. 91, pp. 133-141, 2018.

[14] A. B. Tee, M. D. Bowman, and K. C. Sinha, "A fuzzy mathematical approach for bridge condition evaluation," Civil Engineering Systems, vol. 5, no. 1, pp. 17-24, 1988.

[15] H. Melhem and S. Aturaliya, "Bridge condition rating using an eigenvector of priority settings," Computer-Aided Civil and Infrastructure Engineering, vol. 11, no. 6, pp. 421-432, 1996.

[16] S. Sasmal, K. Ramanjaneyulu, S. Gopalakrishnan, and N. Lakshmanan, "Fuzzy logic based condition rating of existing reinforced concrete bridges," Journal of Performance of Constructed facilities, vol. 20, no. 3, pp. 261-273, 2006.

[17] M. T. Liang, J. H. Wu, and C. H. Liang, "Multiple layer fuzzy evaluation for existing reinforced concrete bridges," Journal of Infrastructure Systems, vol. 7, no. 4, pp. 144-159, 2001.

[18] Z. Li, Z. Shi, and E. T. Ososanya, "Evaluation of bridge conditions using artificial neural networks," in Proceedings of the IEEE Southeastcon'96 in Bringing Together Education, Science and Technology, Tampa, FL, USA, April 1996.

[19] J. Cattan and J. Mohammadi, "Analysis of bridge condition rating data using neural networks," Computer-Aided Civil and Infrastructure Engineering, vol. 12, no. 6, pp. 419-429, 1997.

[20] J. O. Sobanjo, "A neural network approach to modeling bridge deterioration," in Proceedings of Computing in Civil Engineering Congress, pp. 623-626, ASCE, Philadelphia, PA, USA, June 1997.

[21] G. Morcous, H. Rivard, and A. M. Hanna, "Modeling bridge deterioration using case-based reasoning," Journal of Infrastructure Systems, vol. 8, no. 3, pp. 86-95, 2002.

[22] Y. M. Wang and T. Elhag, "Evidential reasoning approach for bridge condition assessment," Expert Systems with Applications, vol. 34, no. 1, pp. 689-699, 2008. 
[23] X. Deng, Y. Hu, and Y. Deng, "Bridge condition assessment using D numbers," Scientific World Journal, vol. 2014, Article ID 358057, 11 pages, 2014.

[24] S. Moufti, T. Zayed, and S. A. Dabous, "Defect-based condition assessment of concrete bridges: fuzzy hierarchical evidential reasoning approach," Transportation Research Record: Journal of the Transportation Research Board, vol. 2431, no. 1, pp. 88-96, 2014.

[25] T. W. Ryan, R. A. Hartle, J. E. Mann, and L. J. Danovich, Bridge Inspector's Reference Manual, Report No. FHWA NHI (2006): 03-001, https://www.dot.state.mn.us/bridge/pdf/insp/ birm/birmchapt0-cover.pdf.

[26] T. D. Everett, P. Weykamp, H. A. Capers et al., Bridge Evaluation Quality Assurance in Europe, No. FHWA-PL-08016, 2008.

[27] BRIAR, Bridge Ratings, Inspections and Records Manual, Department of Transportation State of Colorado, Colorado, USA, 2012, https://www.codot.gov/business/consultants/ advertised projects/2014/copy_of_template-interview/sowreferenced-documents- 4 .

[28] AASHTO, Guide Manual for Bridge Element Inspection, American Association of State Highway and Transportation Officials, Washington, DC, USA, 2011.

[29] J. Roberts and R. Shepard, "Bridge management for the 21st century," Transportation Research Record: Journal of the Transportation Research Board, vol. 1696, pp. 197-203, 2000.

[30] R. M. Ellis and P. D. Thompson, "Bridge asset valuation and the role of the bridge management system," in Proceedings of 2007 Annual Conference and Exhibition of the Transportation Association of Canada: Transportation-An Economic Enabler, Canada, October 2007.

[31] A. Dempster, "Upper and lower probabilities induced by a multivalued mapping," Annals of Mathematical Statistics, vol. 38, no. 2, pp. 325-339, 1967.

[32] G. Shafer, A Mathematical Theory of Evidence, Vol. 42, Princeton University Press, Princeton, NJ, USA, 1976.

[33] J. B. Yang and D. L. Xu, "On the evidential reasoning algorithm for multiple attribute decision analysis under uncertainty," IEEE Transactions on Systems, Man, and Cybernetics-Part A: Systems and Humans, vol. 32, no. 3, pp. 289-304, 2002.

[34] J. B. Yang and D. L. Xu, "Nonlinear information aggregation via evidential reasoning in multiattribute decision analysis under uncertainty," IEEE Transactions on Systems, Man, and Cybernetics-Part A: Systems and Humans, vol. 32, no. 3, pp. 376-393, 2002.

[35] J. Yang, "Rule and utility based evidential reasoning approach for multiattribute decision analysis under uncertainties," European Journal of Operational Research, vol. 131, no. 1, pp. 31-61, 2001. 


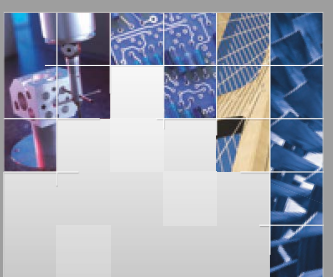

\section{Enfincering}
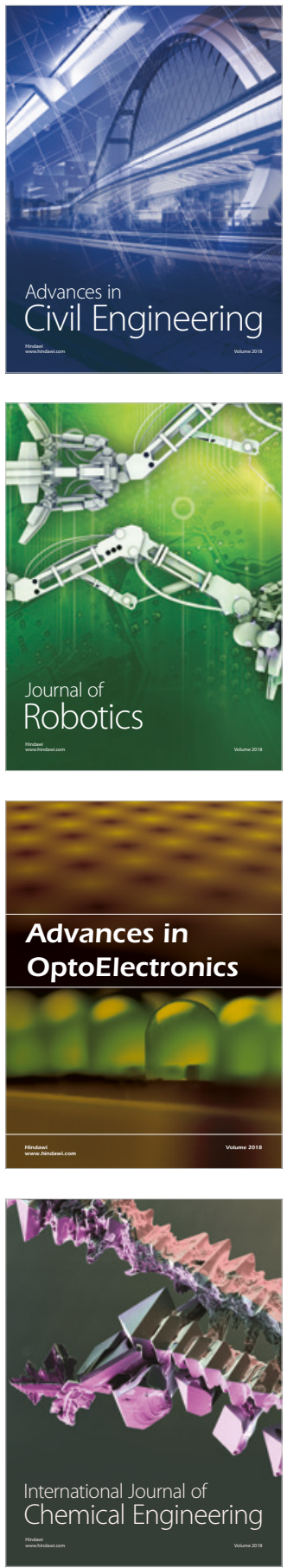

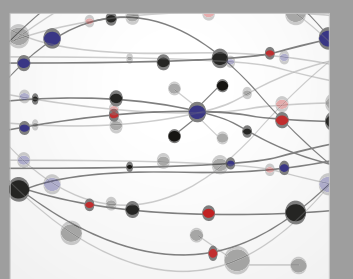

\section{Rotating \\ Machinery}

The Scientific World Journal

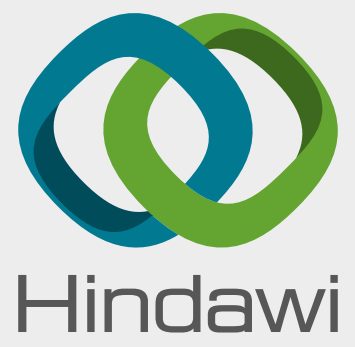

Submit your manuscripts at

www.hindawi.com
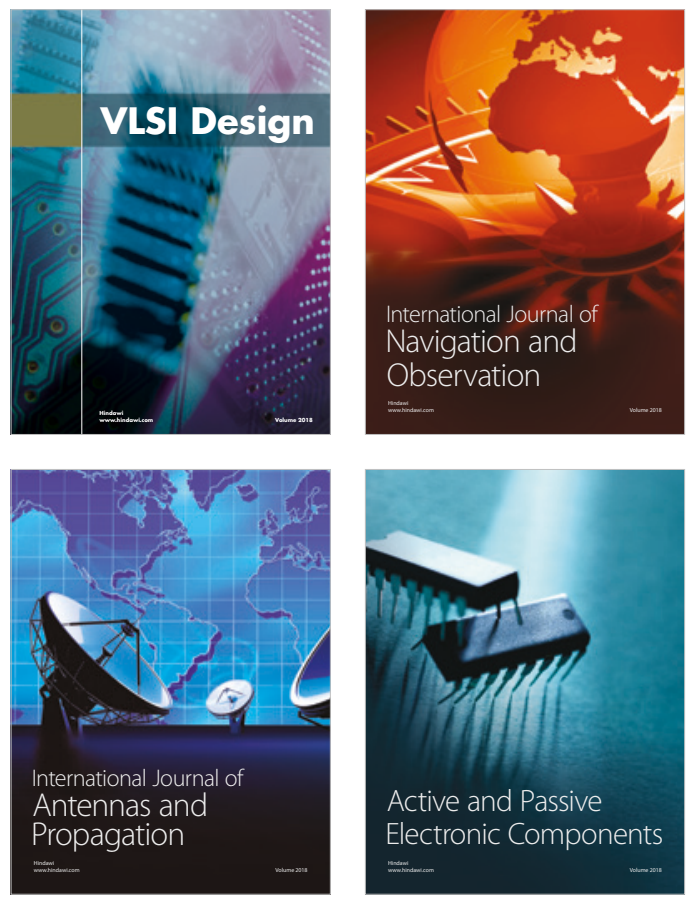
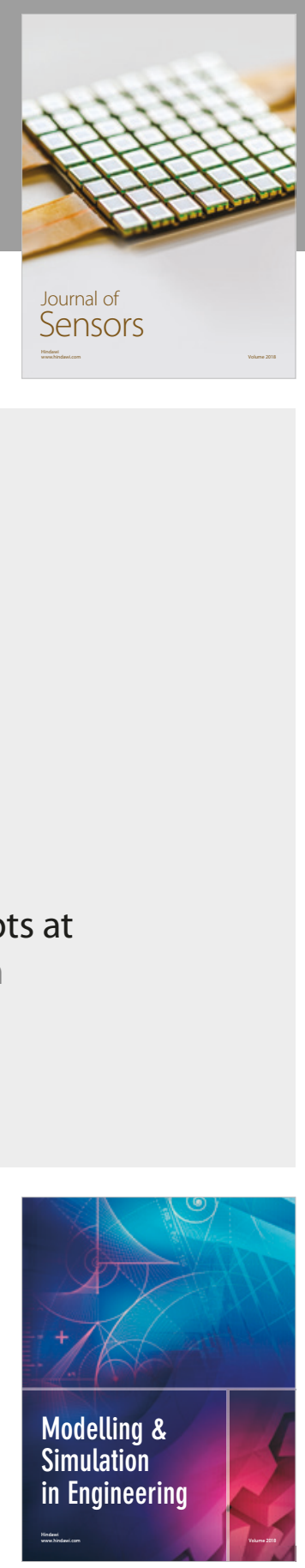

\section{Advances \\ Multimedia}
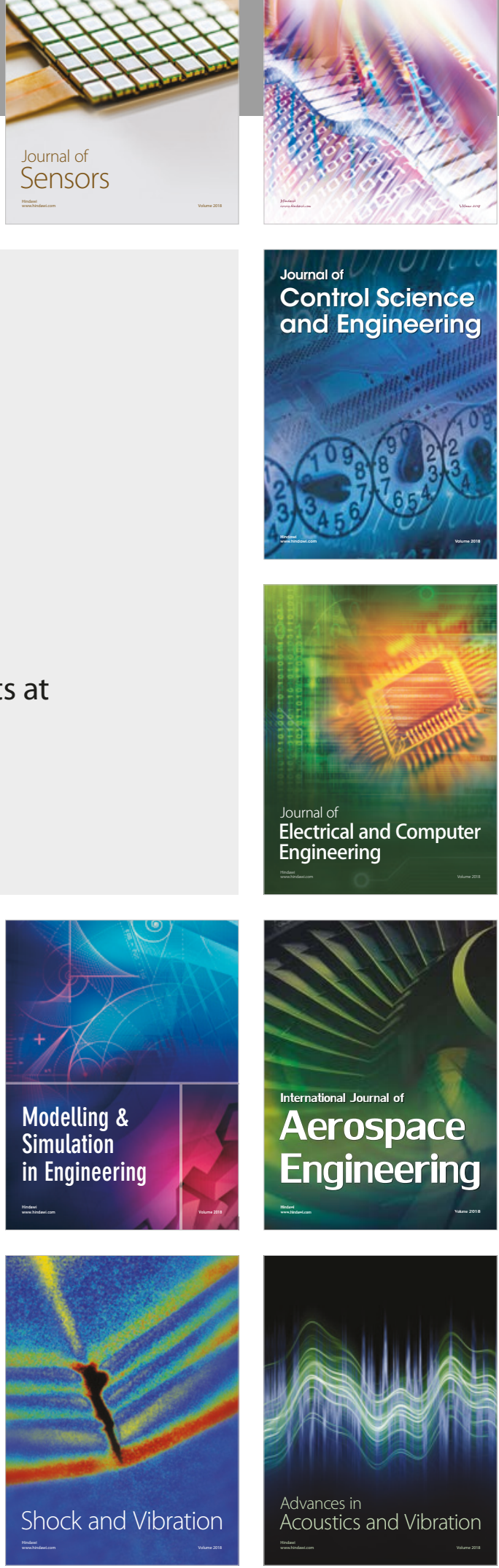